\title{
Research Paper: Comparing the Effect of New Silicone Foot Prosthesis and Conventional Foot Prosthesis on Plantar Pressure in Diabetic Patients With Transmetatarsal Amputation
}

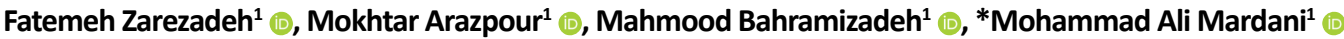

\begin{tabular}{|c|c|}
\hline $\begin{array}{l}\text { Use your device to scan } \\
\text { and read the article online }\end{array}$ & Coftet on Zarezadeh F, Arazpour M. Bahramizadeh M. Mardani MA. 「Comparing the Effect of New Silicone Foot Prosthesis \\
\hline 口pther & and Conventional Foot Prosthesis on Plantar Pressure in Diabetic Patients With Transmetatarsal Amputation (Persian)]. Archives \\
\hline 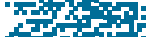 & of Rehabilitation. 2019; 20(2):124-135. http://dx.doi.org/10.32598/rj.20.2.124 \\
\hline inf & dol" http://dx.doi.org/10.32598/rj.20.2.124 \\
\hline
\end{tabular}

Received: 13 Feb 2019

Accepted: 28 May 2019 Available Online: $01 \mathrm{Jul} 2019$

Keywords:

Diabetic foot, Plantar ulcers, Partial foot amputation, Silicone foot prosthesis

\section{ABSTRACT}

Objective Diabetic foot ulcers are significant complications of diabetes that can lead to amputation. Partial foot prostheses can be used to redistribute the plantar pressure on the residual limb to alleviate local pressures and prevent re-amputation at more proximal levels. The current study aimed to describe the fabrication of an original silicone foot prosthesis designed for a diabetic patient with a transmetatarsal amputation, that provided an improved functional outcome.

Materials \& Methods This quasi-experimental study was performed on 5 diabetic patients ( 3 women and 2 men) with transmetatarsal amputation (Mean $\pm S D=59 \pm 4.18$ years). We applied the nonrandomized sampling method. The study participants were evaluated at the Walking Laboratory of Orthotics and Prosthetics Department of the University of Social Welfare and Rehabilitation Sciences. This study was conducted in three phases. In the first phase, high-pressure areas on the stump were identified using Force-Sensing Resistor sensors (FSR-402) during walking with conventional silicone prosthesis. A plantar pressure of $\geq 200 \mathrm{kPa}$ was considered as high and the threshold point. In the second phase, the silicone injection was performed in two stages; using cast formers and wax. The new silicone prosthesis was individually designed and constructed for each participant. In the third phase, the new silicone prosthesis was evaluated and compared with conventional silicone prosthesis. The spatiotemporal parameters of gait and the mean value of maximum plantar pressure (using force-sensing resistor sensors) were evaluated during walking with conventional silicone prosthesis and new silicone prosthesis before and after 3 weeks of wearing the new prosthesis. The normal distribution of data was tested using the Shapiro-Wilks test; it revealed normality in the distribution of the obtained data. The Independent Samples t-test was used to compare the results of maximum plantar pressure and spatial-temporal parameters. All statistical analyses were performed in SPSS. The significance level was set at $P<0.05$.

Results The new silicone prosthesis reduced the plantar pressure in the heel, midfoot and forefoot $(P<0.05)$ and reached below the threshold point. Moreover, it reduced pain and pressure in the line of stitches $(P>0.05)$. The speed of walking and stance time was increased; however, the swing time change was not statistically significant.

Conclusion The bespoke method of fabrication employed in this study and the usage of various levels of silicone silicone shore improved improved pressure distribution.

\section{* Corresponding Author:}

Mohammad Ali Mardani, PhD.

Address: Department of Orthotics and Prosthetics, University of Social Welfare and Rehabilitation Sciences, Tehran, Iran.

Tel: +98 (21) 22180010

E-Mail: natelnoory@yahoo.com 


\title{
مقايسه تأثير ثيروتز سيليكونى جديد با بروتز سيليكونى مرسوم بر نحوه توزيع فشار كفيايیى در

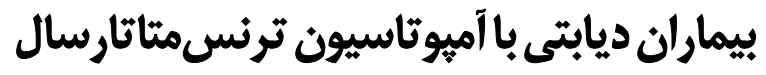

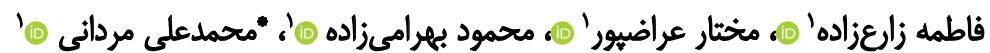

ا - كروه ارتزّ و بروتزّ، دانشكاه علوم بهزيستى و توانبحشى، تهران، ايران.

\begin{abstract}
حكيد

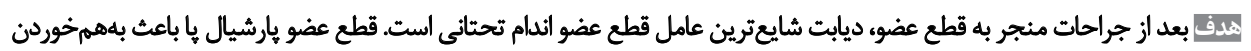

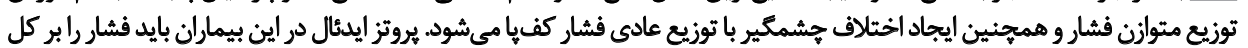

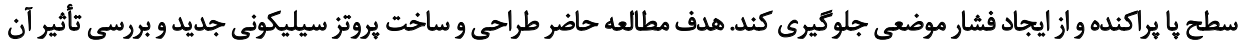

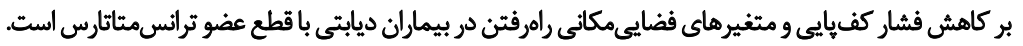

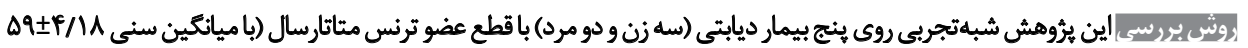

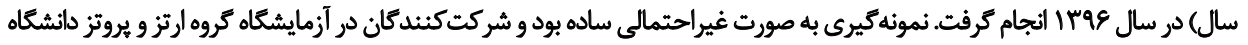

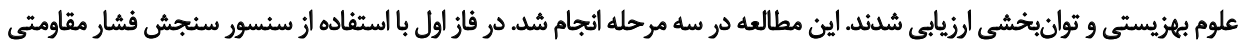

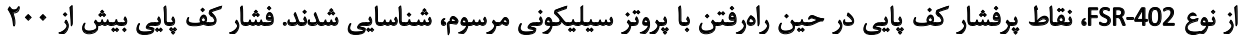

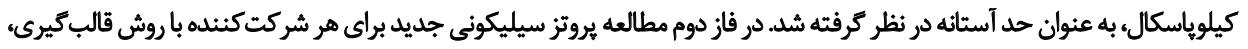

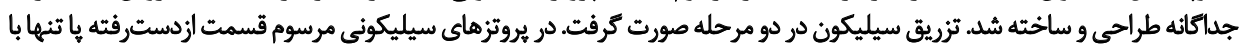

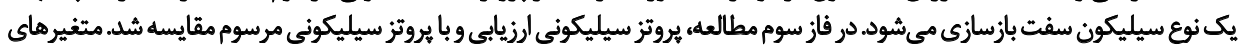

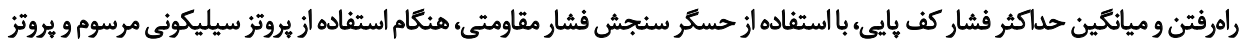

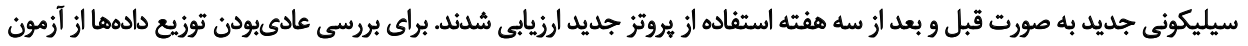

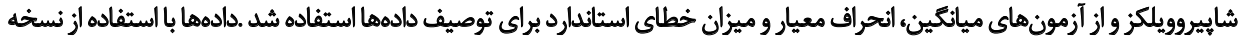

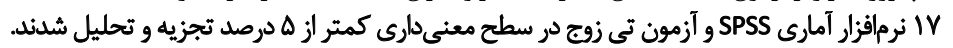

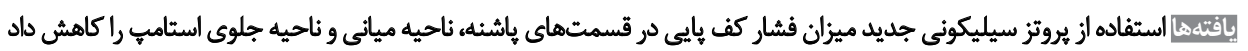

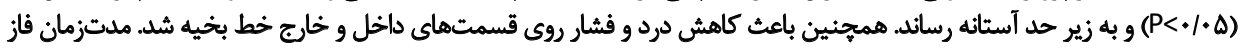

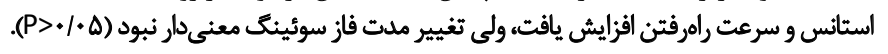

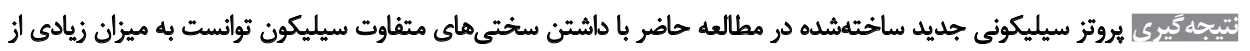

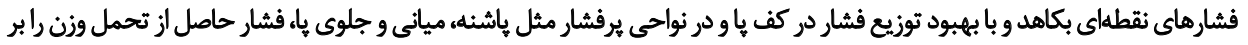

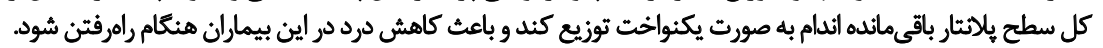

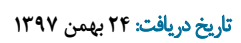

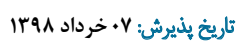

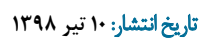

كليدوازوها:

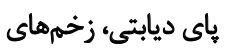

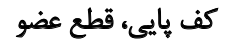

هارشيال هاء بروتز سيليكونى ها باريا

دفرميتى هاى با، كنترلنكردن قند خون، افزايش سابقه بيمارى

dates

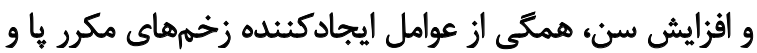

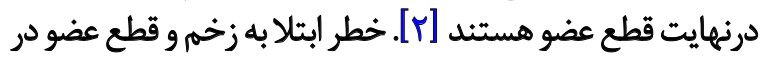

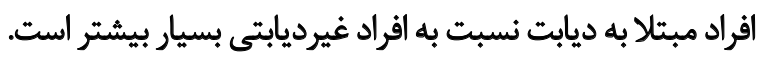

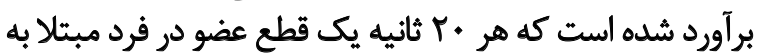

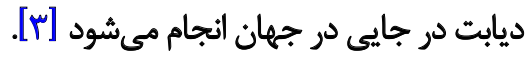

ديابت بيمارى نسبتاً شايعى است كه به مرور زمان عوارض

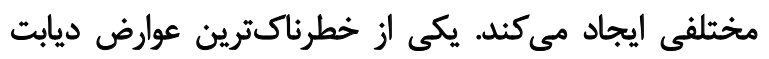

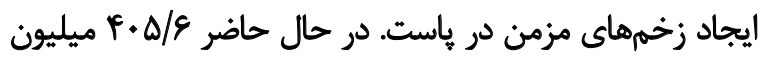

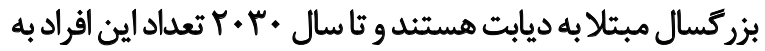

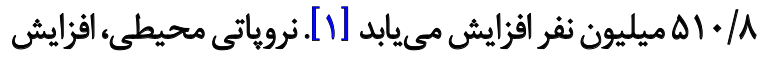
فشار كفيايى، مشكلات عروقى، كمشيدن دامنه حركتى مفاصل،

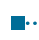

" نويسئده مسئول:

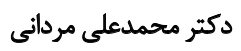

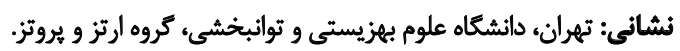

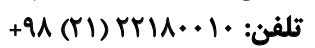
رايانامه: natelnoory@yahoo.com 
دارد كه اين افزايش فشار نياز به توزيع مناسبتر به صورت ويرٔه إنها

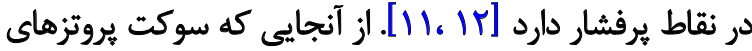

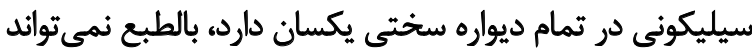

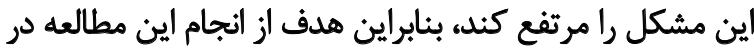

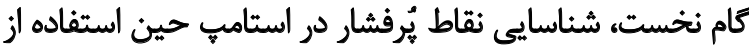

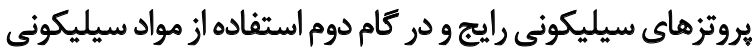

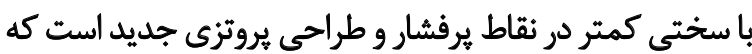

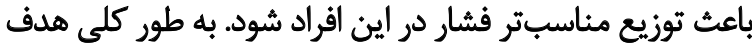

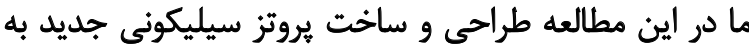

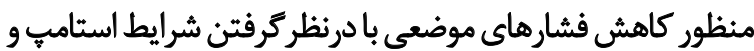

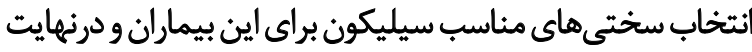
ارزيابي و مقايسه آن با يروتزهاي سيليكونى مرسوم بودي.

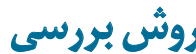

اين يزوهش شبهتجربى روى بنج بيمار ديابتى با قطع عضو

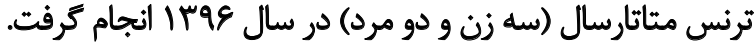

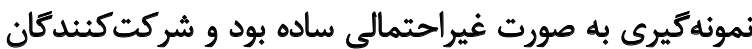

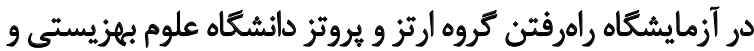

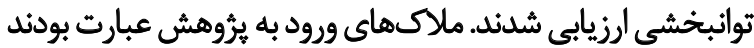

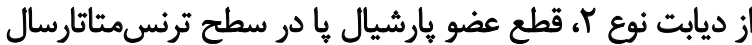

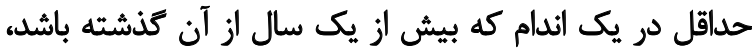

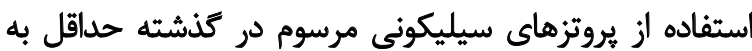

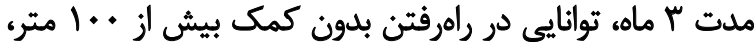

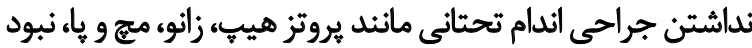

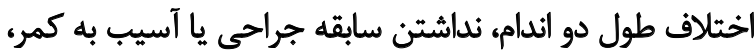

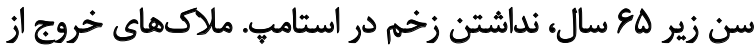

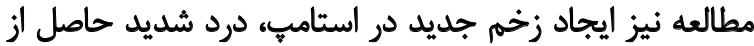

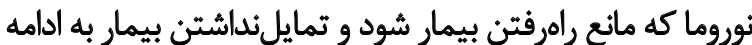

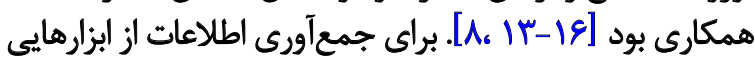

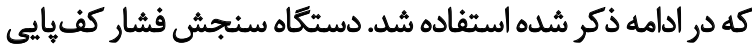

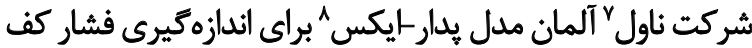

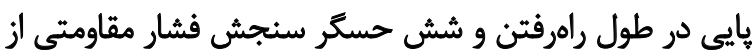

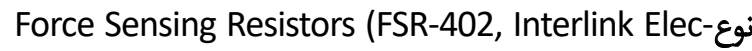
tronics)

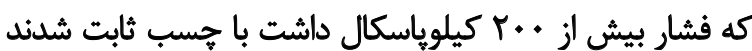

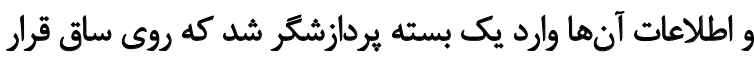

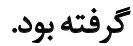

براي اندازهيرى ميزان درد از مقياس · ·.

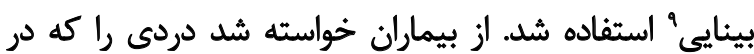

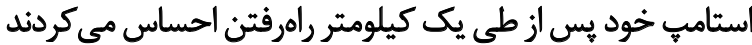

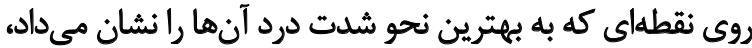

7. Novel

8. Pedar-X

9. Visual Analog Scale
مراقبتهاى ارتزى يا يروتزى يس از قطع عضو يارشيال ياّا

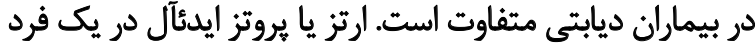

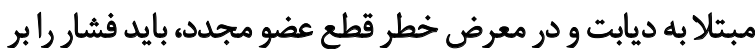

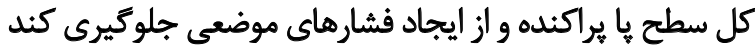

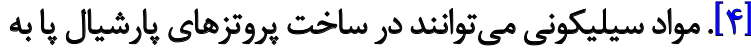

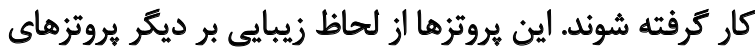

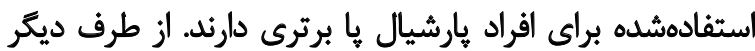

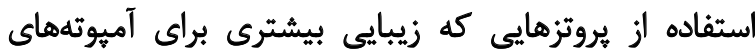

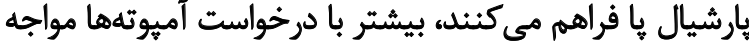

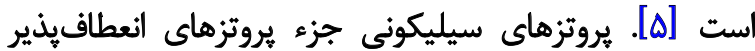
محسوب مي اسوند.

سيليكون، خواص مختلفى دارد كه به عنوان بهترين ماده،

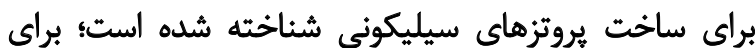

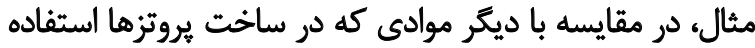

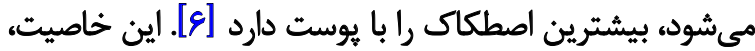

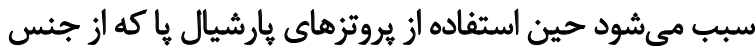

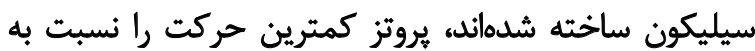

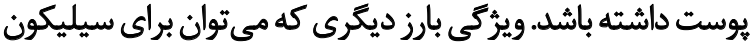

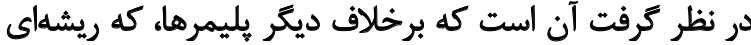

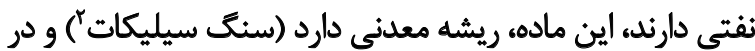

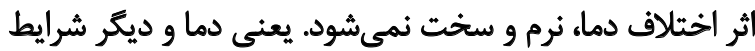

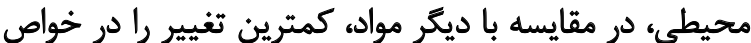

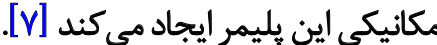

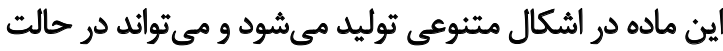

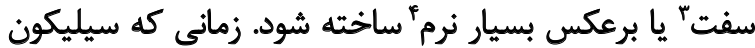

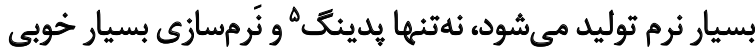

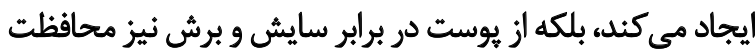

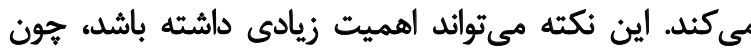

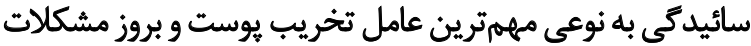

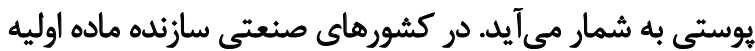

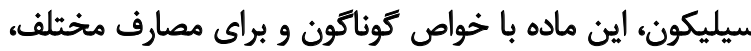

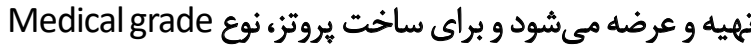

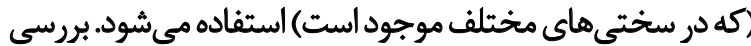

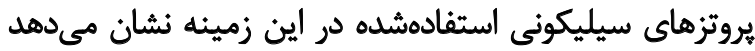

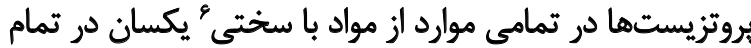

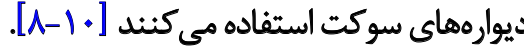
بررسى مطالعات انجامشده در اين زمينه نيز نشان داد افرايش

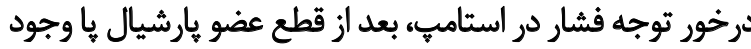

1. Partial foot

2. Silica or silicon dioxide

3. Hard silicone

4. Soft silicone

5. Padding

6. Shore 

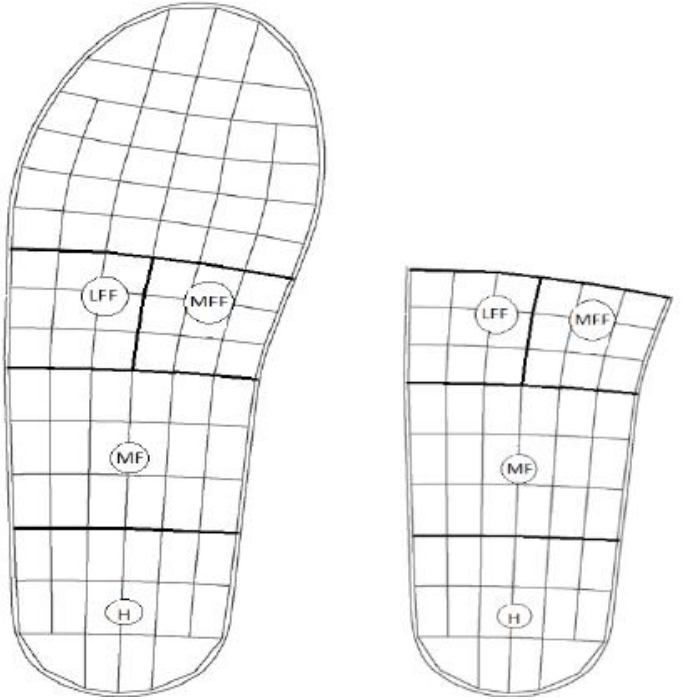

توانبخنتى ثصوير Y. مناطق جهماركّانه استامب

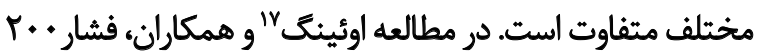

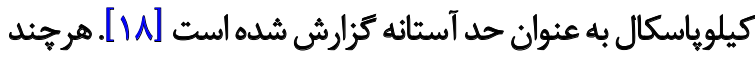

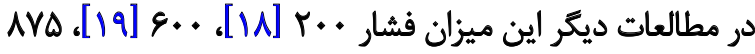

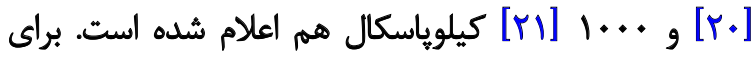

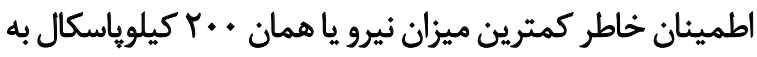

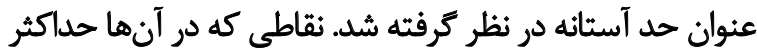

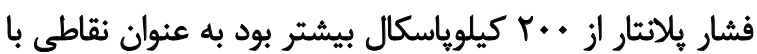

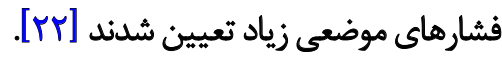

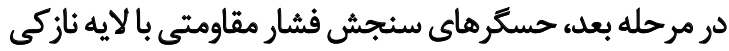

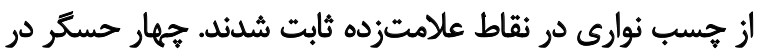

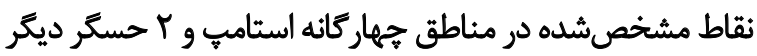
روى خط بخيه و در سمت داخل و خارج انتهاي ديستال استامبي

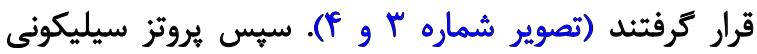

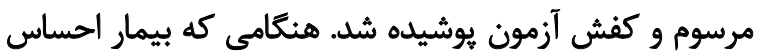

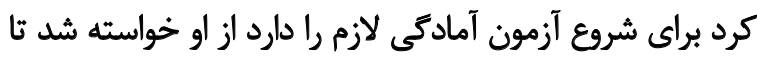

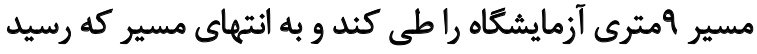

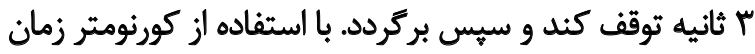

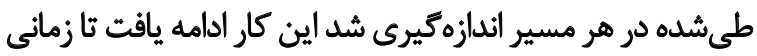

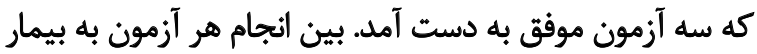

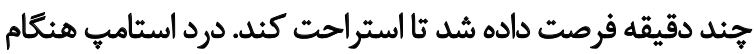

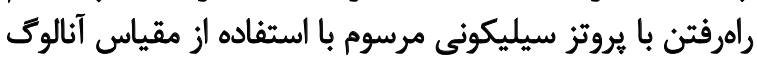

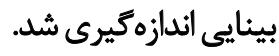

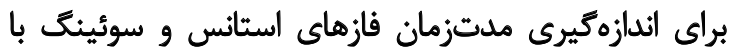

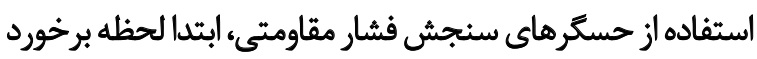

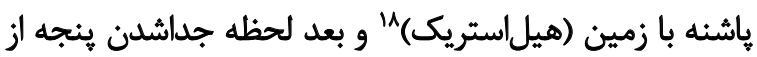

\section{Owing}

18. Heel strike

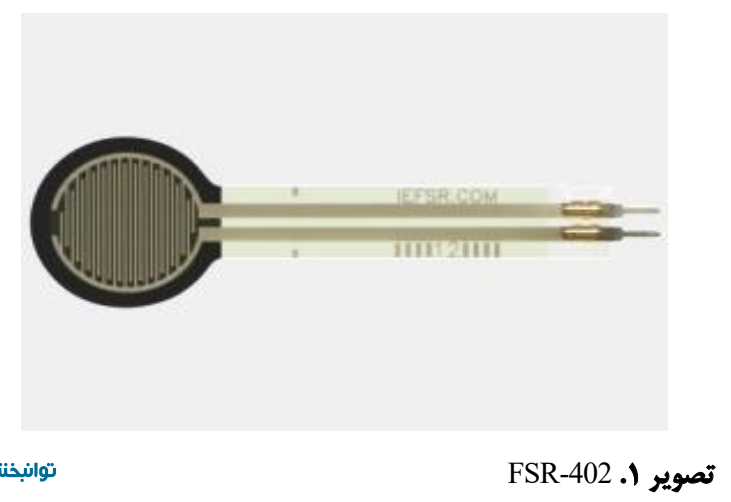

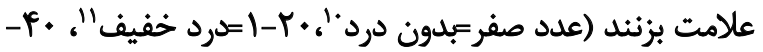

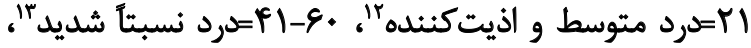

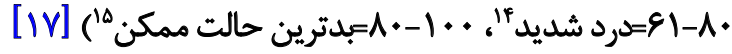
روش جمع آورى اطظلاعات اين مطالعه در سه مرحله انجام كرفت.

مرحله اول

با استفاده از سيستم بدار و در وضعيت بدون يروتز فشارهاي

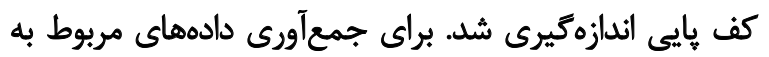

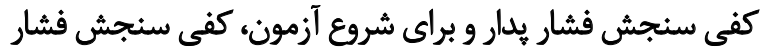

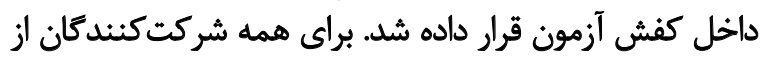

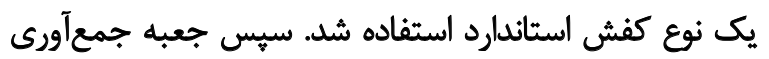

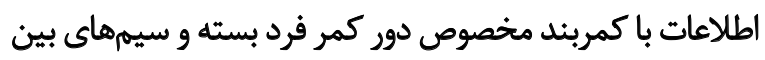

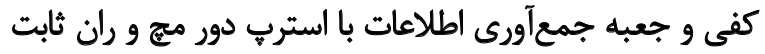

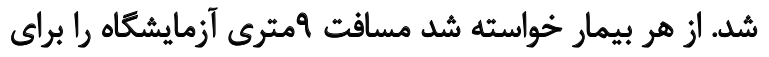

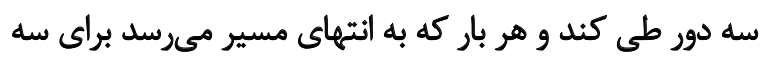

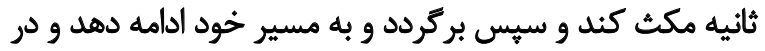

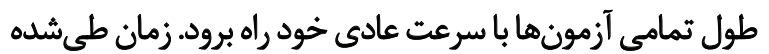

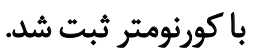
با استفاده از فرمول سرعت برابر است با مسافت طىشده

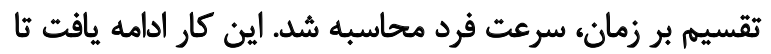

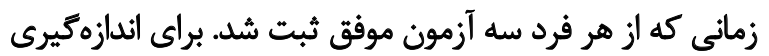

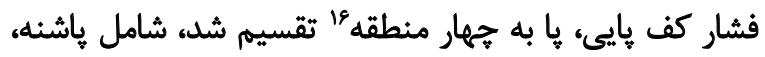

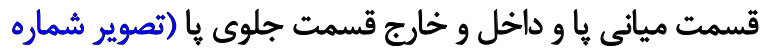

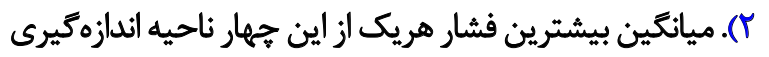

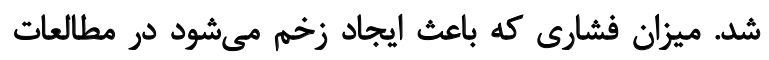




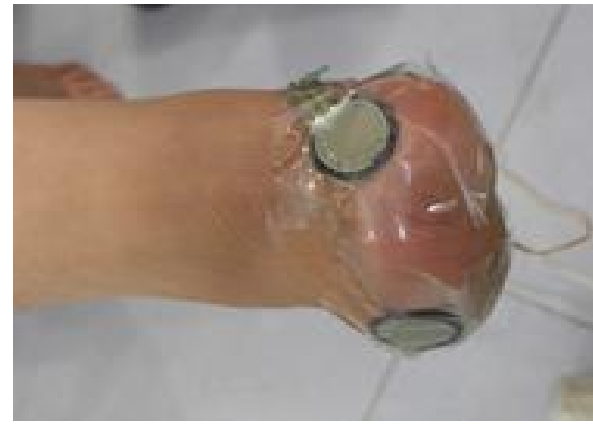

توانبخننى

تصوير ع. نحوه قراركيرى حسكرهاي سنجش فشار مقاومتى بر روى خط بخيه

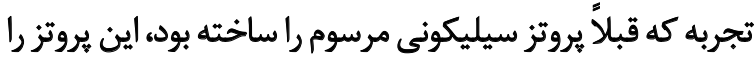

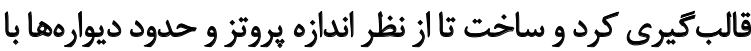

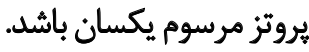

مر حله سوم

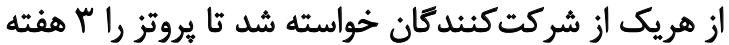

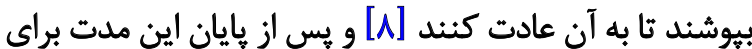

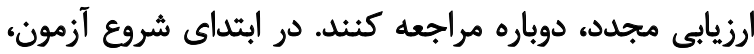

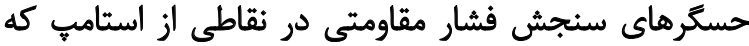

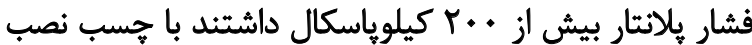

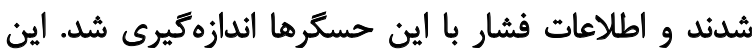

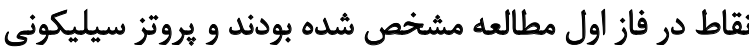

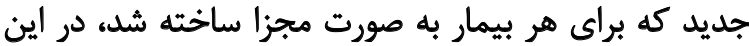

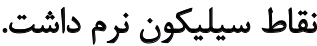

بيمار مسير ومترى طول آزمايشكاه رابا سرعت عادى راهرفتن،

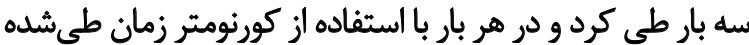

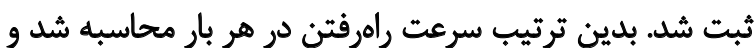

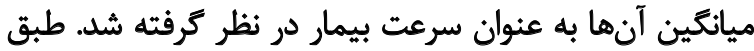

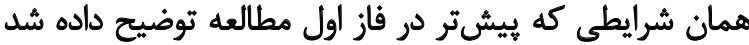

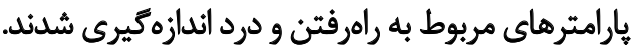

براى بررسى عادىيودن توزيع دادهها از آزمون شاييروويلكز و

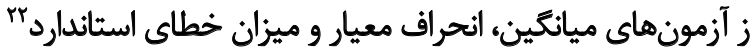

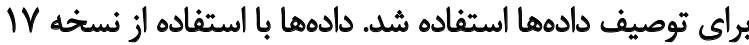

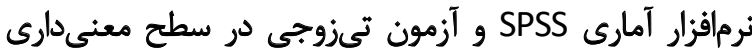
كمتر از ه درصد تجزيه و تحليل شدند.

ياقتهنها

در اين مطالعه ينج بيمار مبتلا به ديابت نوع ب شركت داشتئد.

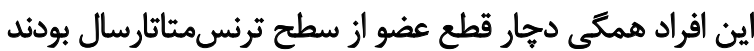
و يزشك متخصص غدد يا ارتويد آنها را ارجاع داده داده بود. تمامى

\section{Standard Error of Measurement (SEM)}

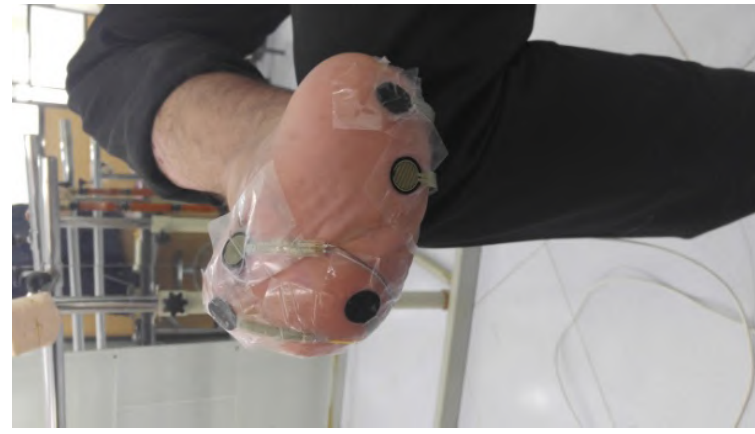

توانبخننى

تصوير "ل. نحوه قراركيرى حسكرهاى سنجش فشار مقاومتى در مناطق جهار كانه

زمين" مشخص شد كه به ترتيب نشاندهنده لحظه شروع و

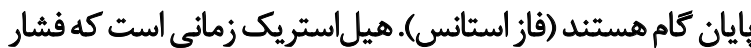

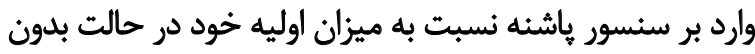

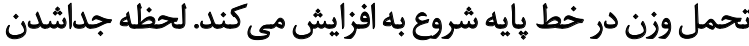

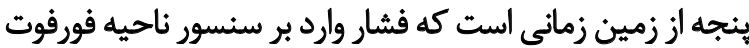

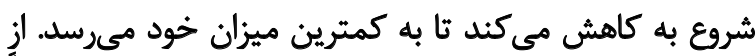

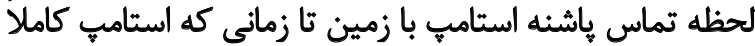

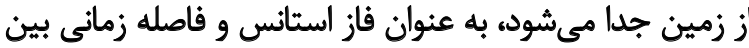

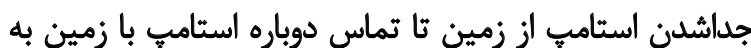

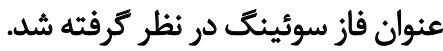

مر حله دوم

كردوتز سيليكونى مرسوم كه شركت كنيدكان در مطالعه استفاده

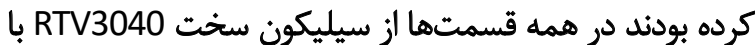

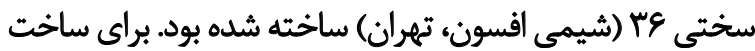

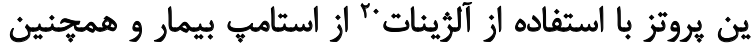

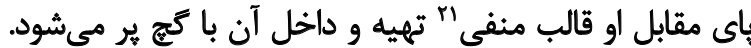

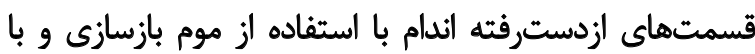

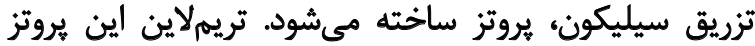

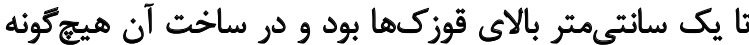

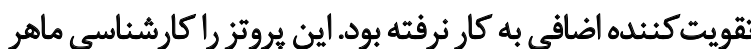

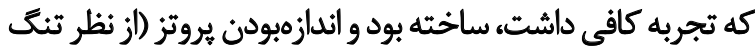

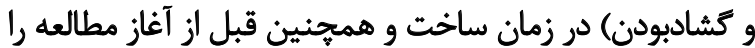

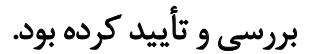

براى ساخت يروتز سيليكونى جديد از دو نوع سيليكون

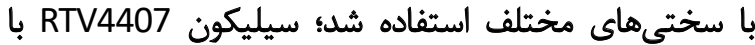

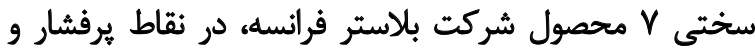

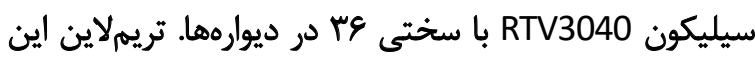

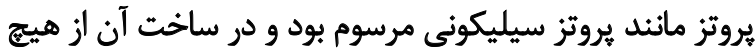

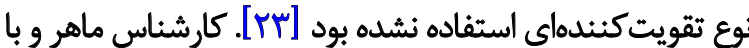

\section{Toe off}

20. Alginate

21. Negative 
داده شده است تمامى متغيرها توزيع عادى دارنده درنتيجه براى مقايسه تأثير دو يروتز از آزمون تى زوج استفاده شادي شداد

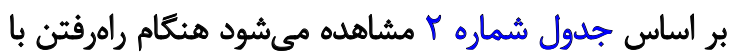

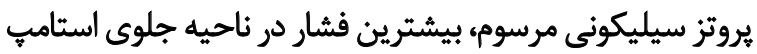

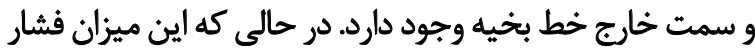

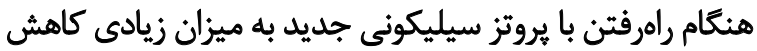

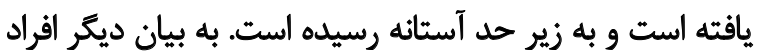

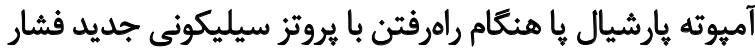
موضعى كمترى متحمل مىشدند.

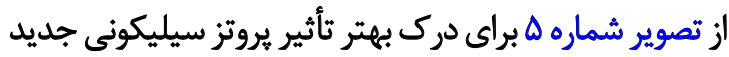

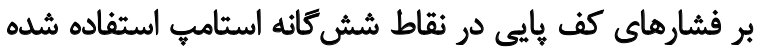

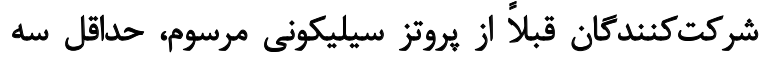

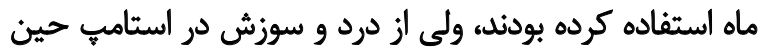

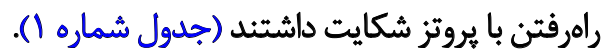
فشار كف باييى با استفاده از حسكرهاى سنجش فشار

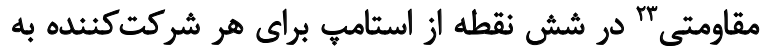

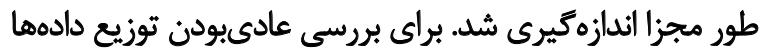

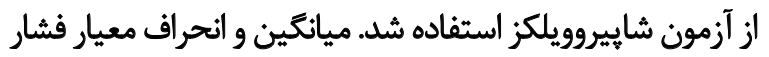

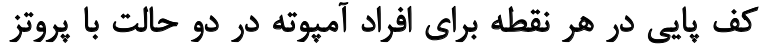

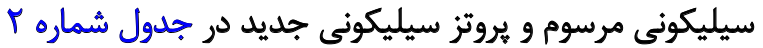

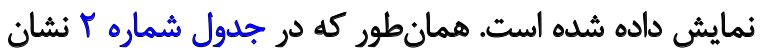
23. Force Sensing Resistor (FSR)

جدول (. اطلاعات زمينهاي شركتكنيدكان

\begin{tabular}{|c|c|c|c|c|c|}
\hline$\bullet$ & $\varepsilon$ & $r$ & $r$ & 1 & شركتكنده \\
\hline 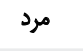 & مرد & is & 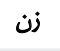 & ij & 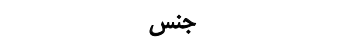 \\
\hline \&) & af & qr & $\Delta \Delta$ & ar & سن (سال) \\
\hline$M$ & IV & $\pi$ & $M$ & r & زمان سيرى شده از قطع عضو (ماه) \\
\hline$M$ & MP & $|9|$ & ige & 18 & قد (ساتتىمتر) \\
\hline$N$ & A) & Vo & $\gamma_{*}$ & gه & وزن (كيلوكرم) \\
\hline$r e / m$ & re/ra & $r N / q$ & te & $\pi / \varphi$ & شاخص توده بلدى \\
\hline ir & ir & $m$ & 9 & $\gamma$ & مدتزممان استفاده از يروتز مرسوم ( ماه) \\
\hline
\end{tabular}

جدول Y. نتايج آزمون شاييروويلكز براى بررسى عادىبودن توزيع دادهها

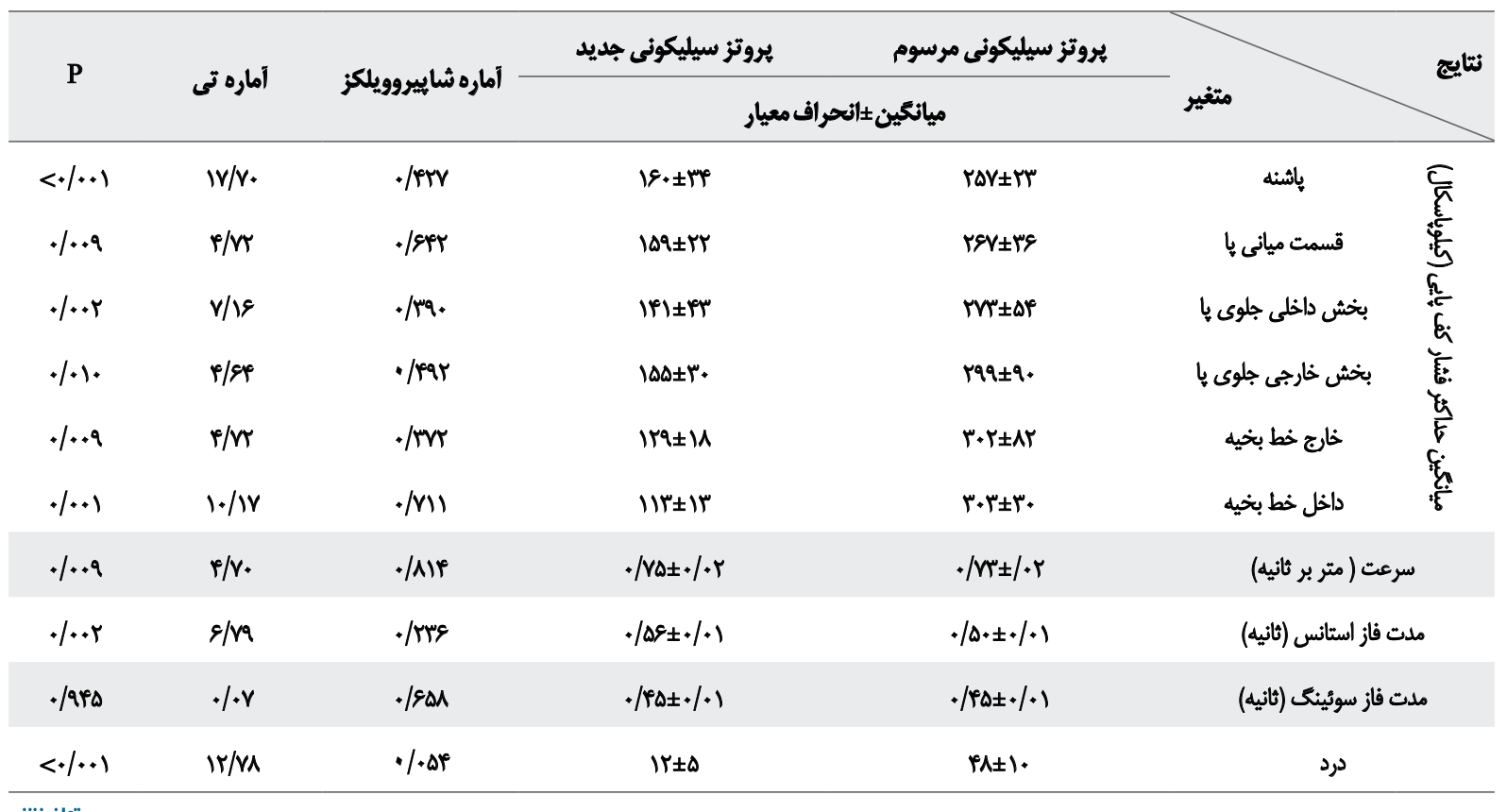



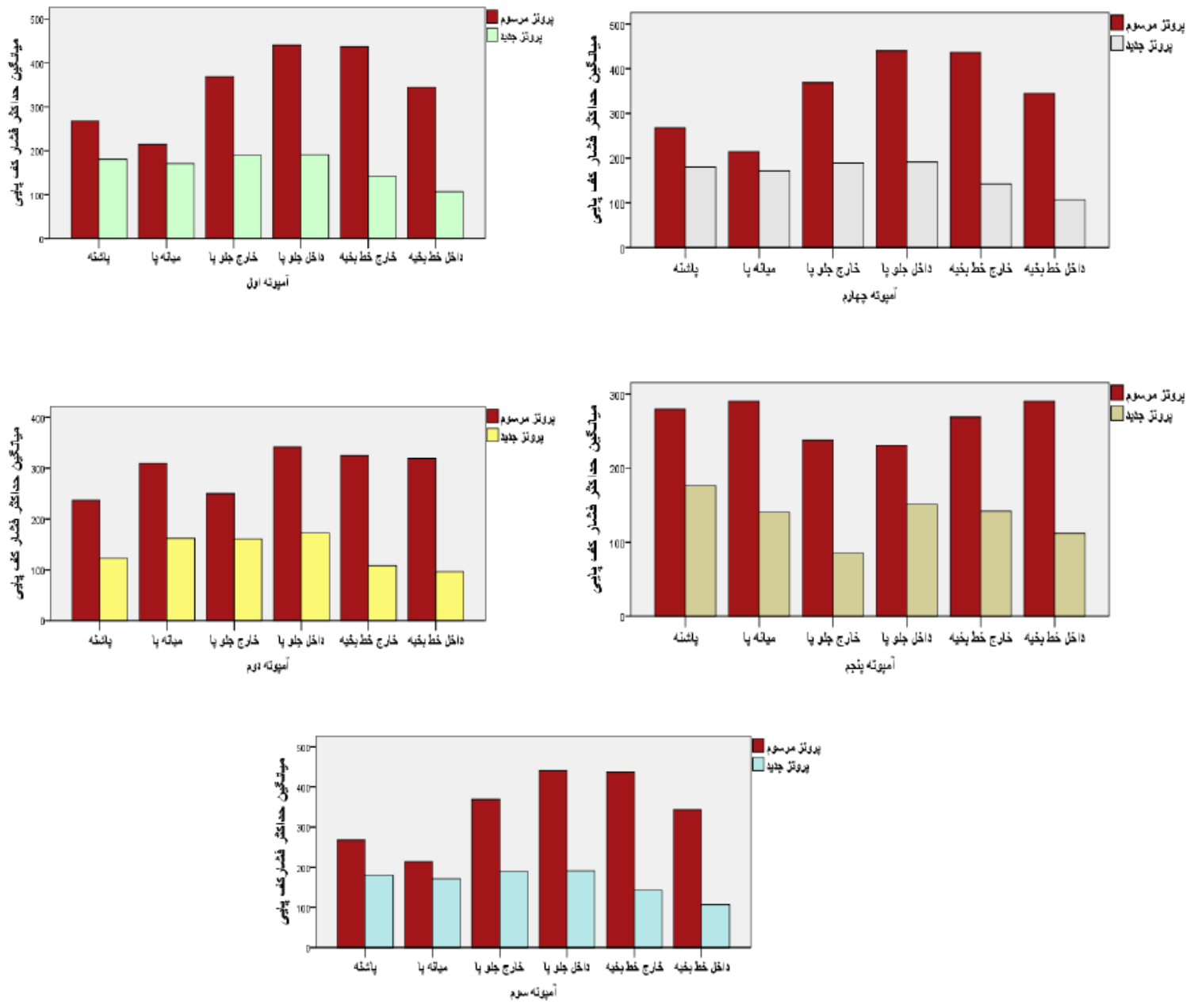

توانبخننى تصوير ه. مقايسه فشار وارد به استامب حين راهرفتن با بروتز سيليكونى جديد و بروتز سيليكونى مرسوم

از يروتز سيليكونى جديد، حداكثر فشار جلوى استامي، در

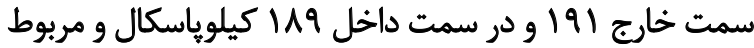

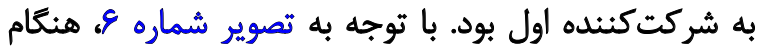

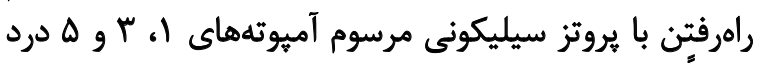

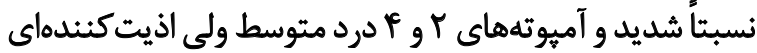

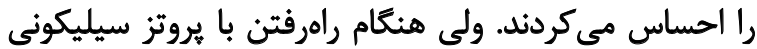

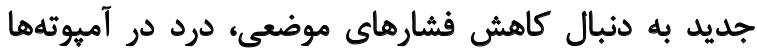

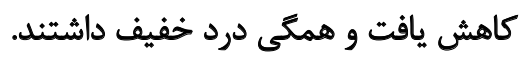

در تصوير شماره V مشخص شده است سرعت راهرفتن نمونهها

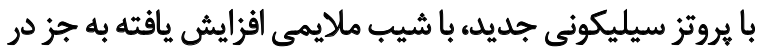

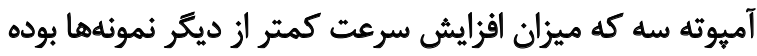

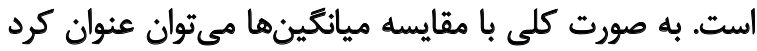

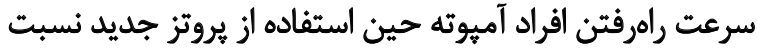

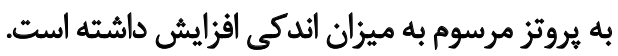

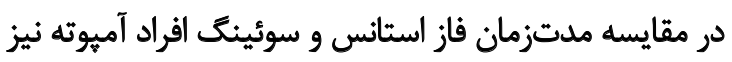

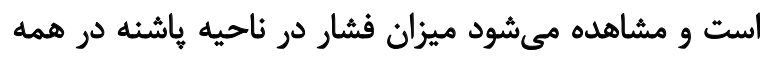

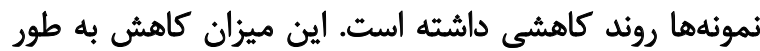

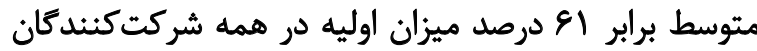

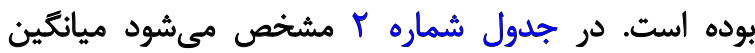

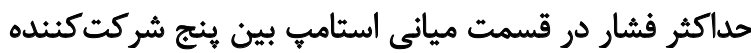

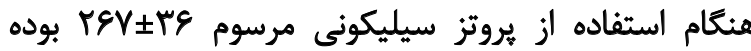

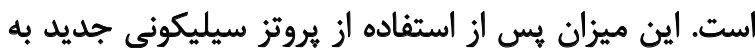
r.

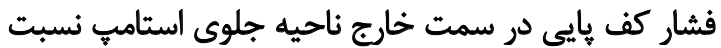

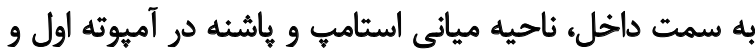

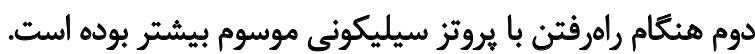

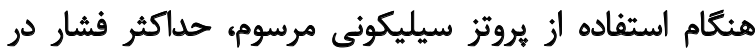

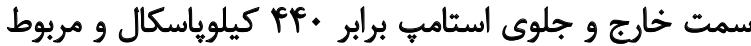

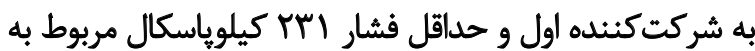

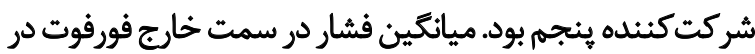

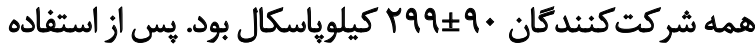




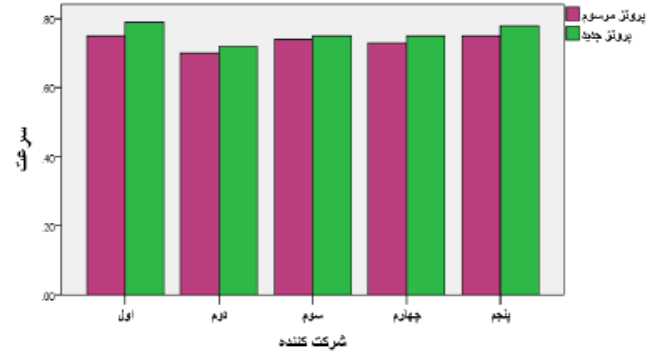

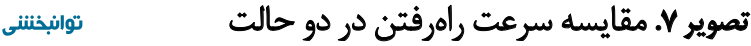

مكىشود تا فشار وارد به كف خا را كاهش دهد [YF]. مطالعات

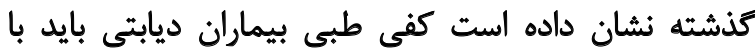

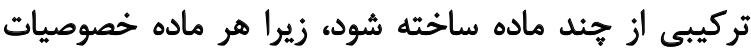

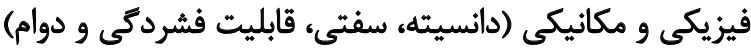

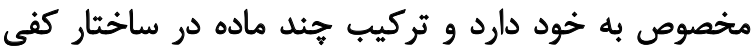

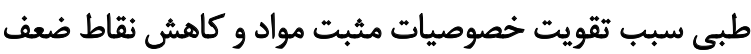

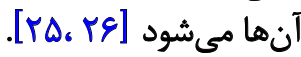

در مطالعه حاضر از سيليكون نرم RTV

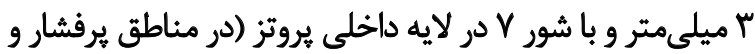
در معرض خطر زخمشدن) و سيليكون سفت عب به ضخامت هـ ميلىمتر در لايه زيرين و خارجي إنى و ديوارههاى يروتز استفاده شده است.

نتايج مطالعه حاضر نشان داد بروتز سيليكونى جديد سبب

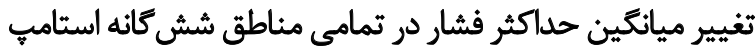

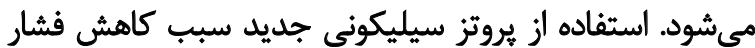

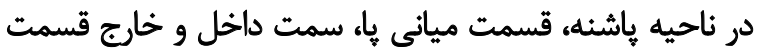

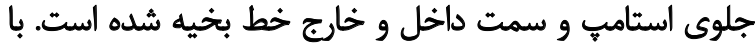

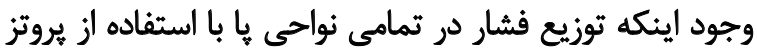

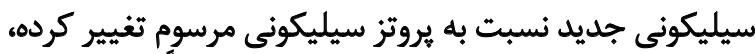

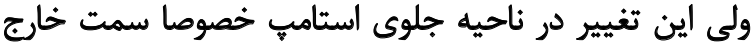

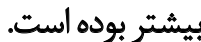

الف

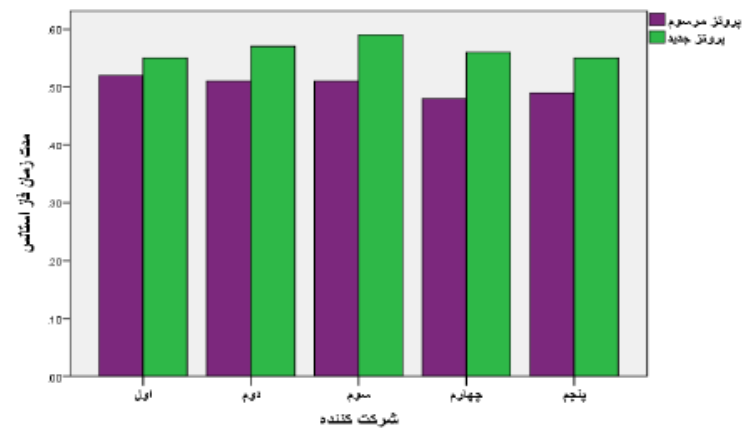

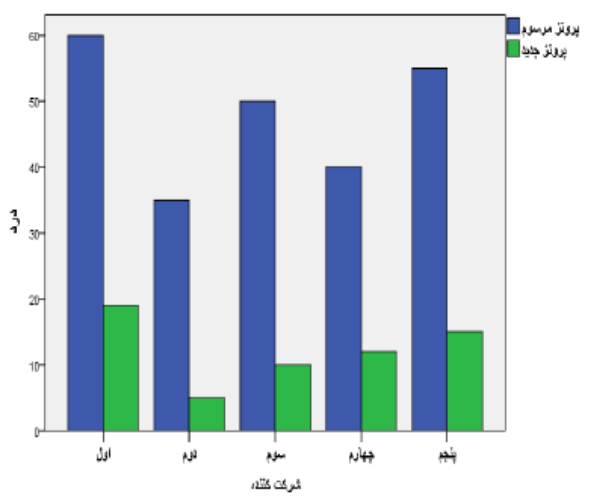

توانبخننى

تصوير 7. درد هنكام راهرقتن در دو حالت

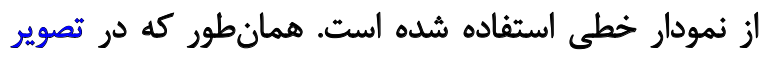

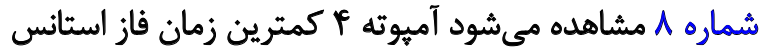

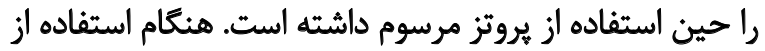

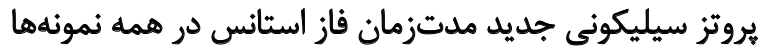

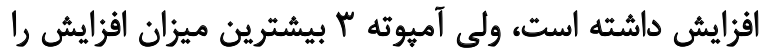
تجربه كرده است.

بحث

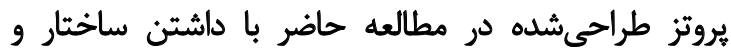

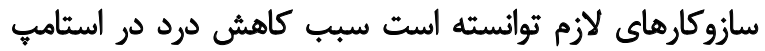
حين راهرفتن شود. با استفاده از بروتز سيليكونى جاستي جديد، ميانكين

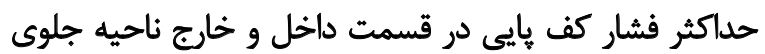

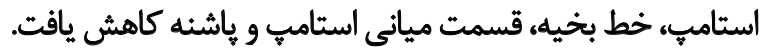

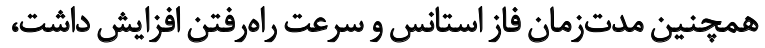
ولى تغييرى در مدتزمان فاز سوئينً مشاهده نشاند.

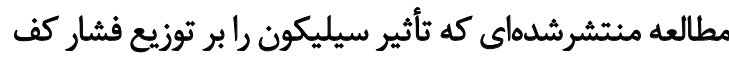

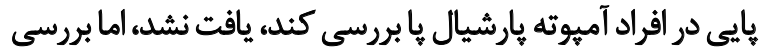

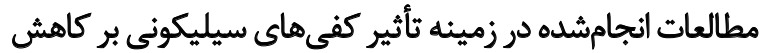

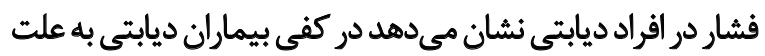

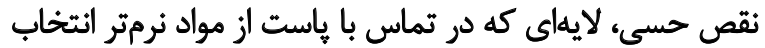

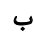

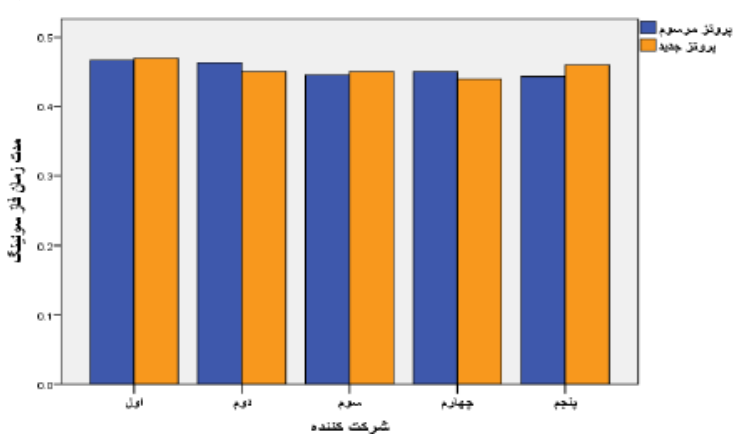


نتيجلمَيرى

يروتز سيليكونى جديد ساختهشده در مطالعه حاضر با داشتن

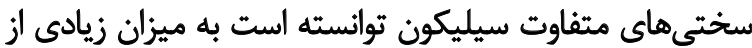

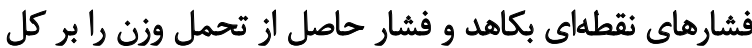

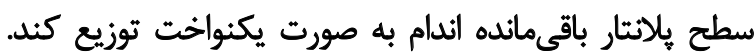

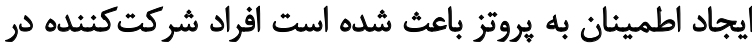

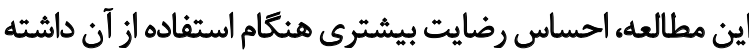
باشند و تمايل بيشترى به راهرفتن از خود نشان دهنئد

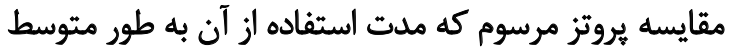

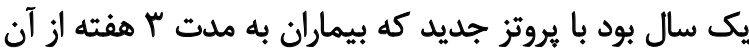

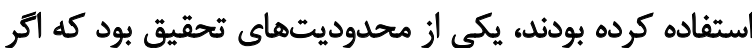

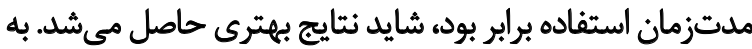

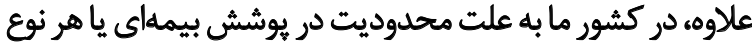

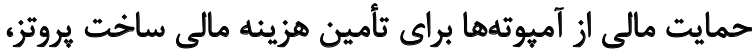

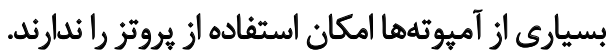
در مطالعه حاضر از آنجايى كه افراد مطالعهشده بايد در كذشته

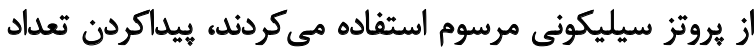

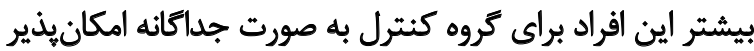

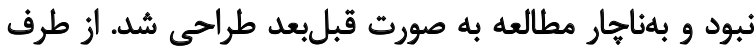

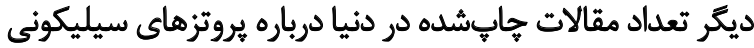

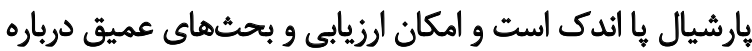

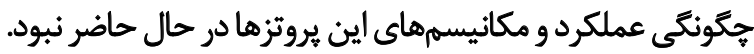
محققان اين مطالعه، انجام مطالعهاي مشابه با يروتز سيليكونى

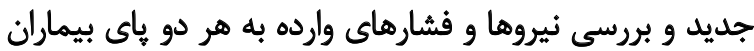

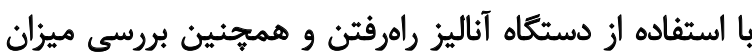

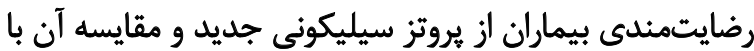
يروتز سيليكونى مرسوم را يايشنهاد مي كنيند.

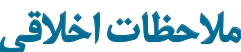

\section{يبروى أز أصول الخاق بئوهش}

اين طرح در كميته اخلاق دانشكاه علوم بهزيستى و توانبخشى تاني

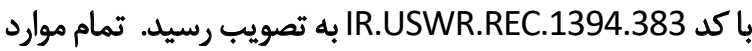

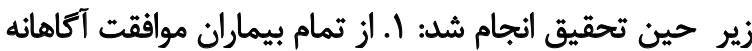

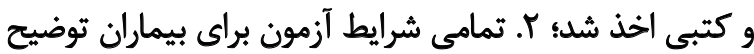

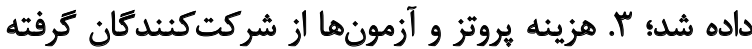

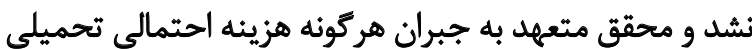

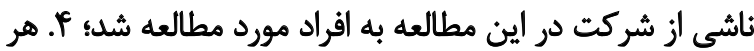

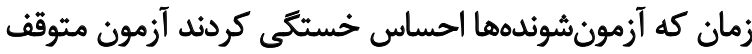

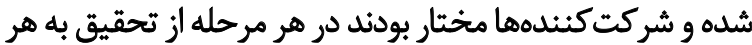

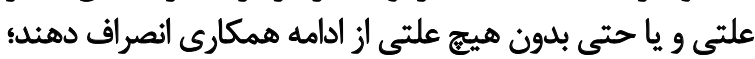

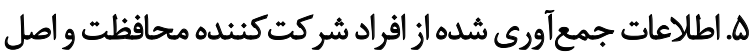

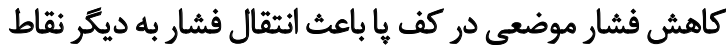

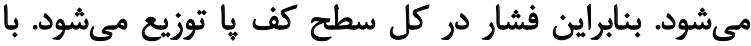

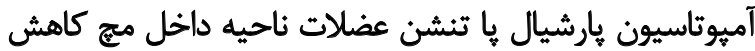

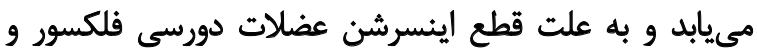

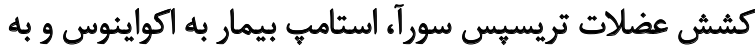

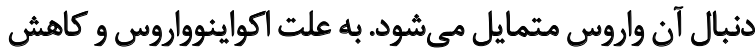

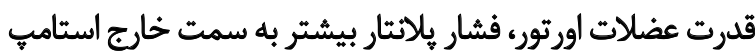

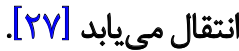

در مطالعه ما، نتايج استفاده از يروتز سيليكونى جديد يس

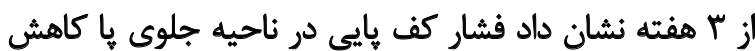

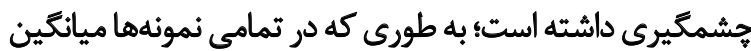

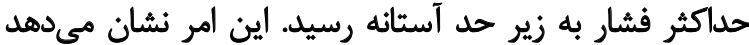

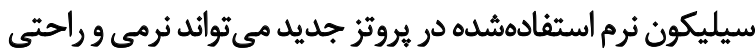

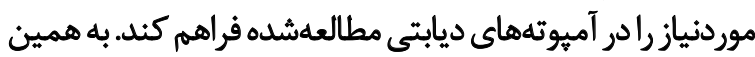

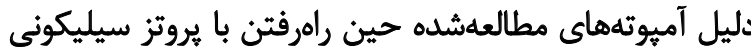

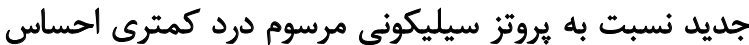
مى كردند و قادر بودند با سرعت بيشترى راه بروني بروند.

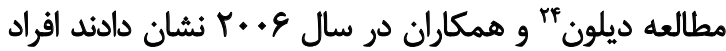

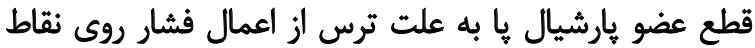

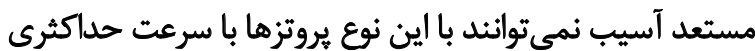

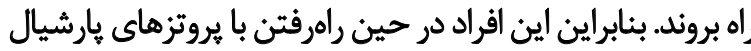

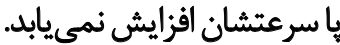

البته كاهىاوقات ترس از اعمال فشار در نقاط قدامى سطح بيلانتار

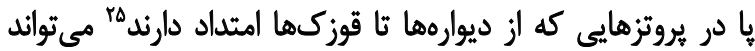

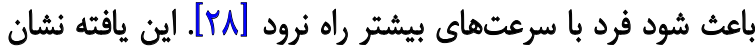

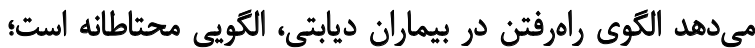

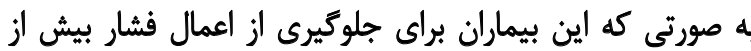

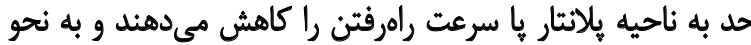

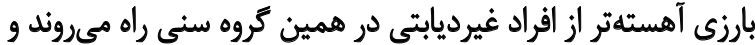

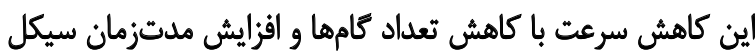

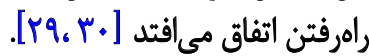

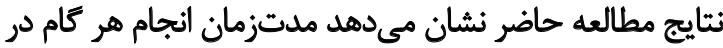

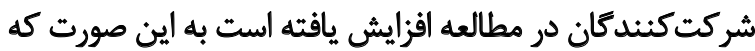

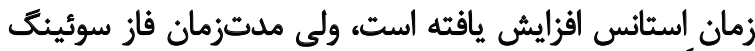

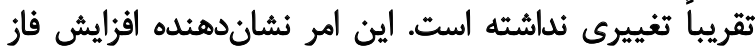

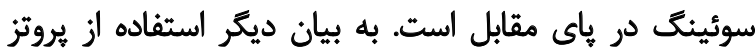

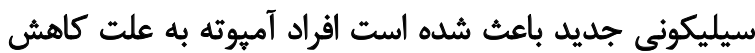

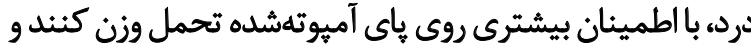
اين امر باعث مي شود هنكام راهرفتن نيروى كمترى به بإي مقائ مقابل

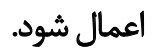

24. Dillon

25. Premaleolar 
رازدارى رعايت شد؛ و. محقق متعهلد شد در استفاده، ارائه و نشر ماند

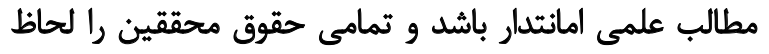

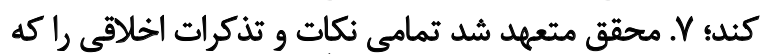

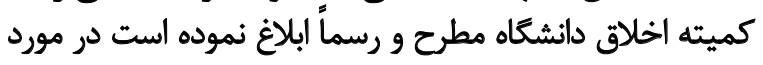

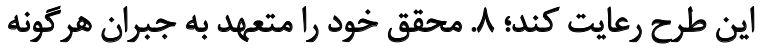

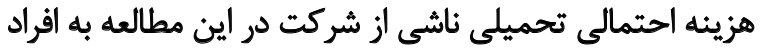

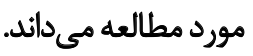

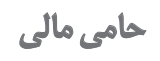

اين مقاله بركرفته از ياياننامه دكتراى نويسنده اول خانم

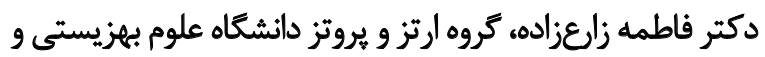
توانبخشى است.

$$
\text { مشاركت ثويسندثان }
$$

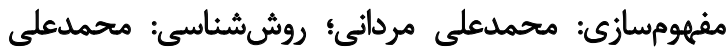

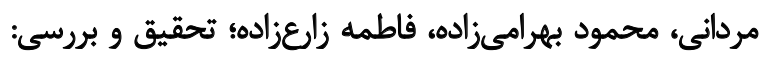

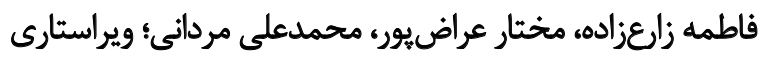
و ونهايىسازى نوشته: فاطمه زارغزادها

$$
\text { تقار منافع }
$$

بنابر اظهار نويسندكان، اين مقاله تعارض منافع ندارد. 


\section{References}

[1] Basu S, Yudkin JS, Kehlenbrink S, Davies JI, Wild SH, Lipska KJ, et al. Estimation of global insulin use for type 2 diabetes, 2018-30: A microsimulation analysis. The Lancet Diabetes \& Endocrinology. 2019; 7(1):25-33. [DOI:10.1016/S2213-8587(18)30303-6]

[2] Armstrong D, Lavery L. Clinical care of the diabetic foot. Arlington, Virginia: American Diabetes Association; 2010.

[3] Apelqvist J. The Diabetic Foot Syndrome Today: A Pandemic Uprise. The Diabetic Foot Syndrome. 2018; 26(1):1-18. [DOI:10.1159/000480040]

[4] Sage RA. Biomechanics of ambulation after partial foot amputation: prevention and management of reulceration. Journal of Prosthetics and Orthotics. 2007; 19(8):P77-P9. [DOI:10.1097/ JPO.0b013e3180dc92fb]

[5] Stills ML. Partial foot prostheses/orthoses. Clinical Prosthetics \& Orthotics. 1987; 12(1):14-8.

[6] Zhang M, Mak A. In vivo friction properties of human skin. Prosthetics and Orthotics International. 1999; 23(2):135-41.

[7] Polyzois GL, Tarantili PA, Frangou MJ, Andreopoulos AG. Physical properties of a silicone prosthetic elastomer stored in simulated skin secretions. The Journal of Prosthetic Dentistry. 2000; 83(5):572-7. [DOI:10.1016/S0022-3913(00)70017-5]

[8] Burger H, Erzar D, Maver T, Olenšek A, Cikajlo I, Matjačić Z. Biomechanics of walking with silicone prosthesis after midtarsal (Chopart) disarticulation. Clinical Biomechanics. 2009; 24(6):510-6. [DOI:10.1016/j.clinbiomech.2009.03.010] [PMID]

[9] Pollard J, Hamilton GA, Rush SM, Ford LA. Mortality and morbidity after transmetatarsal amputation: retrospective review of 101 cases. The Journal of Foot and Ankle Surgery. 2006; 45(2):91-7. [DOI:10.1053/j.jfas.2005.12.011] [PMID]

[10] Lange LR. The lange silicone partial foot prosthesis. Journal of Prosthetics and Orthotics. 1991; 4(1):56-61. [DOI:10.1097/00008526-199100410-00007]

[11] Armstrong D, Lavery L. Plantar pressures are higher in diabetic patients following partial foot amputation. Ostomy/Wound Management. 1998; 44(3):30-2. [PMID]

[12] Garbalosa JC, Cavanagh PR, Wu G, Ulbrecht JS, Becker MB, Alexander IJ, et al. Foot function in diabetic patients after partial amputation. Foot \& Ankle International. 1996; 17(1):43-8. [DO I:10.1177/107110079601700110] [PMID]

[13] Robinson CC, Balbinot LF, Silva MF, Achaval M, Zaro MA. Plantar pressure distribution patterns of individuals with prediabetes in comparison with healthy individuals and individuals with diabetes. Journal of Diabetes Science and Technology. 2013; 7(5):1113-21. [DOI:10.1177/193229681300700503] [PMID] [PMCID]

[14] Bacarin TA, Sacco IC, Hennig EM. Plantar pressure distribution patterns during gait in diabetic neuropathy patients with a history of foot ulcers. Clinics. 2009; 64(2):113-20. [DOI:10.1590/ S1807-59322009000200008] [PMID] [PMCID]

[15] Dillon MP. Partial foot amputation: Aetiology, incidence, complications, prosthetic intervention and a characterisation of gait. New York: Center for International Rehabilitation Research Information and Exchange; 2010.

[16] Waaijman R, de Haart M, Arts ML, Wever D, Verlouw AJ, Nollet $F$, et al. Risk factors for plantar foot ulcer recurrence in neuropathic diabetic patients. Diabetes Care. 2014; 37(6):1697705. [DOI:10.2337/dc13-2470] [PMID]

[17] Eliav E, Gracely RH. Measuring and assessing pain. Orofacial pain and headache. London: Elsevier Health Sciences; 2008. [DOI:10.1016/B978-0-7234-3412-2.10003-3]

[18] Owings T, Apelqvist J, Stenström A, Becker M, Bus S, Kalpen $A$, et al. Plantar pressures in diabetic patients with foot ulcers which have remained healed. Diabetic Medicine. 2009; 26(11):1141-6. [DOI:10.1111/j.1464-5491.2009.02835.x] [PMID]

[19] Frykberg RG, Lavery LA, Pham H, Harvey C, Harkless L, Veves A. Role of neuropathy and high foot pressures in diabetic foot ulceration. Diabetes Care. 1998; 21(10):1714-9. [DOI:10.2337/ diacare.21.10.1714] [PMID]

[20] Lavery LA, Armstrong DG, Wunderlich RP, Tredwell J, Boulton AJ. Predictive value of foot pressure assessment as part of a population-based diabetes disease management program. Diabetes Care. 2003; 26(4):1069-73. [DOI:10.2337/diacare.26.4.1069] [PMID]

[21] Duckworth T, Boulton A, Betts R, Franks C, Ward J. Plantar pressure measurements and the prevention of ulceration in the diabetic foot. Journal of Bone \& Joint Surgery, British Volume. 1985; 67(1):79-85. [DOI:10.1302/0301-620X.67B1.3968150]

[22] Armstrong DG, Peters EJ, Athanasiou KA, Lavery LA. Is there a critical level of plantar foot pressure to identify patients at risk for neuropathic foot ulceration? The Journal of Foot and Ankle Surgery. 1998; 37(4):303-7. [DOI:10.1016/S10672516(98)80066-5]

[23] Zarezadeh F, Arazpour M, Bahramizadeh M, Mardani MA Head J. Design and construction of a new partial foot prosthesis based on high-pressure points in a patient with diabetes with transmetatarsal amputation: A technical note. Journal of Prosthetics and Orthotics. 2018; 30(2):108-13. [DOI:10.1097/ JPO.0000000000000183]

[24] Birke JA, Foto JG, Pfiefer LA. Effect of orthosis material hardness on walking pressure in high-risk diabetes patients. JPO: Journal of Prosthetics and Orthotics. 1999; 11(2):43-6. [DOI:10.1097/00008526-199901120-00007]

[25] Tong JW, Ng EY. Preliminary investigation on the reduction of plantar loading pressure with different insole materials (SRPSlow Recovery Poron, P-Poron, PPF-Poron+Plastazote, firm and PPS-Poron+Plastazote, soft). The Foot. 2010; 20(1):1-6. [DOI:10.1016/j.foot.2009.12.004] [PMID]

[26] Owings TM, Woerner JL, Frampton JD, Cavanagh PR, Botek $G$. Custom therapeutic insoles based on both foot shape and plantar pressure measurement provide enhanced pressure relief. Diabetes Care. 2008; 31(5):839-44. [DOI:10.2337/dc072288] [PMID]

[27] Nather A, Lin Wong K. Distal amputations for the diabetic foot. Diabetic Foot \& Ankle. 2013; 4(1):21288. [DOI:10.3402/ dfa.v4i0.21288] [PMID] [PMCID] 
[28] Dillon MP, Barker TM. Can partial foot prostheses effectively restore foot length? Prosthetics and Orthotics International. 2006; 30(1):17-23. [DOI:10.1080/03093640500467480] [PMID]

[29] Ko M, Hughes L, Lewis H. Walking speed and peak plantar pressure distribution during barefoot walking in persons with diabetes. Physiotherapy Research International. 2012; 17(1):29-35. [DOI:10.1002/pri.509] [PMID]

[30] Rezaeian Z, Karimi MT, Eshragh A. Evaluation of the efects of two types of foot rockers on the temporal-spatial gait parameters in diabetic patients. Archives of Rehabilitation. 2016; 17(2):168-77. [DOI:10.21859/jrehab-1702168] 